Aus der Landesforschungsanstalt für Landwirtschaft und Fischerei Mecklenburg-Vorpommern, Institut für Tierproduktion Dummerstorf ${ }^{\prime}$ und dem Forschungsinstitut fur die Biologie landwirtschaflicher Nutztiere, Forschungsbereich Fortpflanzungsbiologie ${ }^{2}$, Dummerstorf

\author{
FRANK REHBOCK', FALK SCHNEIDER ${ }^{2}$, WOLFGANG ZUPP'
} und SVEN GRUMBACH'

\title{
Saisonale Einfliusse auf die Konzentration von Progesteron im Blutplasma von Müttern der Rasse Schwarzköpfiges Fleischschaf (Kurzmitteilung)
}

\section{Summary}

Title of the paper: Seasonal influences on blood plasma progesterone concentration in ewes of Black head Mutton sheep breed (short communication)

In 71 Black head mutton sheeps on pasturing and housing were determined from the year 1995 to 1997 and there in the period from March to October the concentration of blood progesterone for determination of onset of estrus. The level of blood progesterone reported, on literature also, no cycle on this period. The investigation of 6 of this sheeps with ultrasonography had the result of ovarian activity with follicles from $2 \mathrm{~mm}$ to $7 \mathrm{~mm}$ in diameter, but no cycle was determined.

The progesterone level of minimum and maximum on all years differed from March to beginning of September between $0,33 \mathrm{ng} / \mathrm{ml}$ and $2,15 \mathrm{ng} / \mathrm{ml}$ blood progesterone, after this to October between $0,82 \mathrm{ng} / \mathrm{ml}$ and $4,25 \mathrm{ng} / \mathrm{ml}$ blood progesterone.

The pregnant sheeps detected blood progesterone concentrations from $5,50 \mathrm{ng} / \mathrm{ml}$ to $13,55 \mathrm{ng} / \mathrm{ml} \mathrm{blood}$ progesterone (twins). The corpus luteum activity may be is prevalent on the blood progesterone level up $2 \mathrm{ng} / \mathrm{ml}$ and pregnancy up $5 \mathrm{ng} / \mathrm{ml}$ blood progesterone.

The detectability of blood progesterone and supplemented ultrasonography of ovary possess the possibility recording of onset cycle activity and early pregnancy diagnosis.

Key words: progesteron, ovarian activity, service season, Black head Mutton sheep breed

\section{Zusammenfassung}

Bei 71 Schafen der Rasse Schwarzköpfiges Fleischschaf wurde von 1995 bis 1997 nacheinander im Zeitraum von März bis Oktober die Progesteronkonzentration im Blutserum zur Feststellung des Brunstbeginns bestimmt. An 6 Schafen fand zusätzlich eine Ultraschalluntersuchung der Ovarien für Zuordnung von Blutwerten zur Ovaraktivität statt.

Die Progesteronwerte zeigten, auch in Anlehnung an die Literatur, keinen klinisch ablaufenden Zyklus an. Die Ultraschalluntersuchung der Ovarien ergab eine Ovaraktivität anhand der Follikelgroßßen von $2 \mathrm{~mm}$ bis $7 \mathrm{~mm}$, aber keine Ovulationen.

Die Minimal-und Maximalwerte von Progesteron im Gesamtzeitraum bewegten sich von März bis Anfang September zwischen $0,33 \mathrm{ng} / \mathrm{ml}$ bis $2,15 \mathrm{ng} / \mathrm{ml}$ Blutserum, danach bis Oktober zwischen $0,82 \mathrm{ng} / \mathrm{ml}$ bis $4,25 \mathrm{ng} / \mathrm{ml}$ Blutserum. Die nachweislich als tragend diagnostizierten Schafe wiesen Progesteronwerte von $5,50 \mathrm{ng} / \mathrm{ml}$ bis $13,55 \mathrm{ng} / \mathrm{ml}$ Blutserum auf (Zwillingsträchtigkeit).

Das Vorkommen der Progesteronkonzentrationen ab $2 \mathrm{ng} / \mathrm{ml}$ Blutserum könnte eine Gelbkörperaktivität und ab $5 \mathrm{ng} / \mathrm{ml}$ eine Trächtigkeit vermuten lassen.

Schlüsselwörter: Progesteron, Ovaraktivität, Decksaison, Schwarzköpfiges Fleischschaf

\section{Einleitung}

In der Schafproduktion vollzog sich in den letzten Jahren eine Umwandlung von der Woll- auf die Lammfleischerzeugung und damit verbunden eine sehr starke Verände- 
rung des Rassespektrums. So gibt es gegenwärtig in Mecklenburg-Vorpommern etwa $80 \%$ Schwarzköpfige Fleischschafe (SKF) und deren Kreuzungen. Dieses SKF ist hauptsächlich saisonal fortpflanzungstauglich. Vorverlagerungen der Bockzeit bis in den August und Juli sind in züchterisch dahingehend bearbeiteten Herden möglich geworden.

Unbedeutend ist bisher der ab März bis Juli in die Bockzeit eintretende Anteil der Schafe.

Für eine kontinuierliche Lammfleischbelieferung des Marktes sind aber Belegungszeiten über längere Zeiträume Bedingung. Durch Selektion, Training und biotechnische Maßnahmen ist die Brunstsaison auf die Sommermonate ausdehnbar.

Auch während des saisonalen Anöstrus ist bei den meisten untersuchten Schafrassen das Verhalten der Follikelentwicklung ähnlich dem während der Zuchtsaison. In diesen Follikelreifungswellen ist weder die Zahl der Antralfollikel noch die der Follikel in ovulatorischer Größe verändert. Doch die Follikelgröße ist als alleiniger Parameter zur qualitativen Beurteilung der Ovarfunktion nicht ausreichend, weil in der Population der Follikel mit ovulatorischer Größe auch östrogeninaktive Follikel enthalten sind, die weniger LH-Rezeptoren als die aktiven enthalten und nicht durch GnRH, LH oder HCG zur Ovulation zu bringen sind (SOUZA u.a., 1996). Die Differenzierung dieser beiden Follikeltypen ist jedoch aufgrund der extrem niedrigen peripheren Östrogenspiegel praktisch nicht möglich.

Ein weiteres Kriterium könnte die Erfassung der LH Pulsfrequenz darstellen, die während des saisonalen Anöstrus durch einen angestiegenen negativen Feedback des Östradiols reduziert ist (GALLEGOS-SANCHEZ u.a., 1998). Diese Bestimmungen erfordern jedoch nicht nur geeignete empfindliche und spezifische Nachweismethoden, sondern setzen auch eine relativ häufige und lückenlose Blutentnahme voraus. Aus diesem Grunde reduzieren sich die derzeitigen hormonanalytischen Möglichkeiten auf das Hormon des Ovargelbkörpers, das Progesteron, dessen quantitative Erfassung bereits in wenigen Punkten bzw. Mehrtagesabständen wertvolle Hinweise zur gesamten Ovarfunktion gibt.

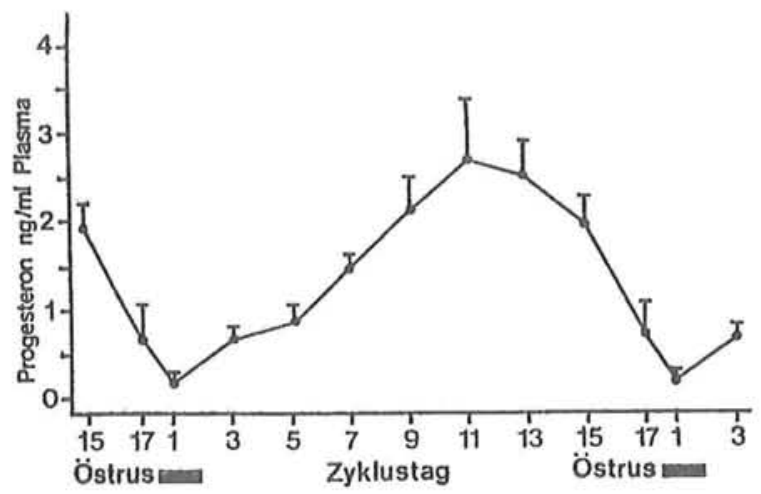

Abb.1: Progesteronverlauf im Sexualzyklus von Schafen (BOSTEDT u.a., 1981) (Concentration of blood progesterone were investigated in average $(\bar{x} \pm s \bar{x})$ of sexual cycle of 5 Merino local sheeps on 12 cycles. Blood samples in two days distance ((BOSTEDT et al., 1981)) 
Die Untersuchung der Progesteronkonzentration im Blutplasma von Mutterschafen in verschiedenen Jahresabschnitten, teilweise unterstützt durch Ultraschalldiagnostik am Ovar, sollte eine Klärung über das Auftreten von Zyklen und der Einflußmöglichkeiten ihrer früheren Nutzung im Jahresverlauf ermöglichen. Über erste Ergebnisse soll nachfolgend berichtet werden.

Der Verlauf der Progesteronkurve in einem vollwertigen Zyklus wird in Abbildung1 dargestellt.

Aus ihm gehen der Konzentrationsbereich, die individuellen Schwankungen im Zyklusverlauf und absoluten Hormonwerte sowie die notwendige Entnahmefrequenz zur sicheren Erfassung von Zyklusminimum (Östruszeitraum)- und -maximum (Gelbkörperphase des Zyklus) hervor.

\section{Material und Methode}

Von 1995 bis 1997 wurde an 71 Schafen der Rasse SKF mit hauptsächlicher Weide und teilweiser Stallhaltung die Progesteronkonzentration im Blutplasma bestimmt. Im Verlauf der Versuchsjahre ist der Zeitraum von März bis Oktober erfaßt worden (Tab.). Zusätzlich wurden bei 6 Schafen die Ovarien mittels Ultraschall von März bis Mai 1997 untersucht, um Zuordnungen von Progesteronwerten und Ovaraktivität zu ermöglichen. Dazu diente das Ultraschallgerät SSD 500 der Fa. „Aloka“ (Japan) und lineare Transrektalsonden mit 5,0 oder 7,5 MHz (Modelle UST $588 \mathrm{U}$ bzw. 5561) sowie ein Drucker der Fa. „Sony“ (UP 860 CE).

Progesteron wurde mit einem bewährten 3 H-Radioimmunoassay (BLÖDOW u.a., $1988)$ in $50 \mu \mathrm{l}$-Aliquoten von Plasmaproben bestimmt, wobei das verwendete Kaninchen-Antiserum weiter gereinigt als Tracer im [1,2,6,7] - 3 H Progesteron (Amersham, Braunschweig) verwendet wurde und zur B/F - Trennung die Dextran - Aktivkohle Methode zum Einsatz kam. Die Tritium Messung erfolgte in einem Flüssigszintillationszähler vom Typ „Spectral“ (Wallace, Finnland), die Auswertung der Titer wurde mit einem integrierten Rechenprogramm vorgenommen.

Tabelle

Tieranzahl und Untersuchungsabschnitte (Number of animals and segments of investigations)

\begin{tabular}{lrccc}
\hline Jahr & $\mathrm{n}$ & Abschnitt & Probenintervall & Standort \\
\hline 1995 & 31 & $4.5 .-2.6$. & 2 Tage & Weide \\
1996 & 13 & $7.5 .-31.7$. & 4 Tage & Weide \\
& 11 & $7.5 .-31.7$. & unregelmäBig & Weide \\
1997 & 6 & $11.3 .-6.5$. & 3 Tage & Stall \\
& 6 & $14.3 .-6.5$. & 4-7 Tage & Stall (Ultraschall) \\
& 2 & $23.9 .-24.9$. & 2 Stunden & Stall \\
& 2 & $25.9 .-30.9$. & täglich & Stall \\
& 1 & $8.10 .-15.10$. & täglich & Stall \\
& 1 & $11.10 .-18.10$. & täglich & Stall \\
& 10 & $26.9 .-17.10$. & 3 Tage & Weide \\
\hline
\end{tabular}

Ergebnisse und Diskussion

Progesterondaten in der Literatur sind gut vergleichbar, weil die methodischen Unter- 
schiede die Aussage zur Gelbkörperaktivität und darüber hinaus zur gesamten Ovarfunktion nur unwesentlich beeinflussen.

Die Erhebung der Progesteronkonzentration 1995 zur Ermittlung von Basiswerten an 31 Schafen von Anfang Mai bis Anfang Juni ergab einen Mittelwert von 1,13 ng/ml Blutplasma mit Extremen von $0,54 \mathrm{ng} / \mathrm{ml}$ bis $2,0 \mathrm{ng} / \mathrm{ml}$ Blutserum (Abb. 2). Dieses Hormonniveau im Verlaufe von vier Wochen deutet auf eine Azyklie hin und ermöglicht demzufolge keine Aussicht auf eine fertile Brunst.

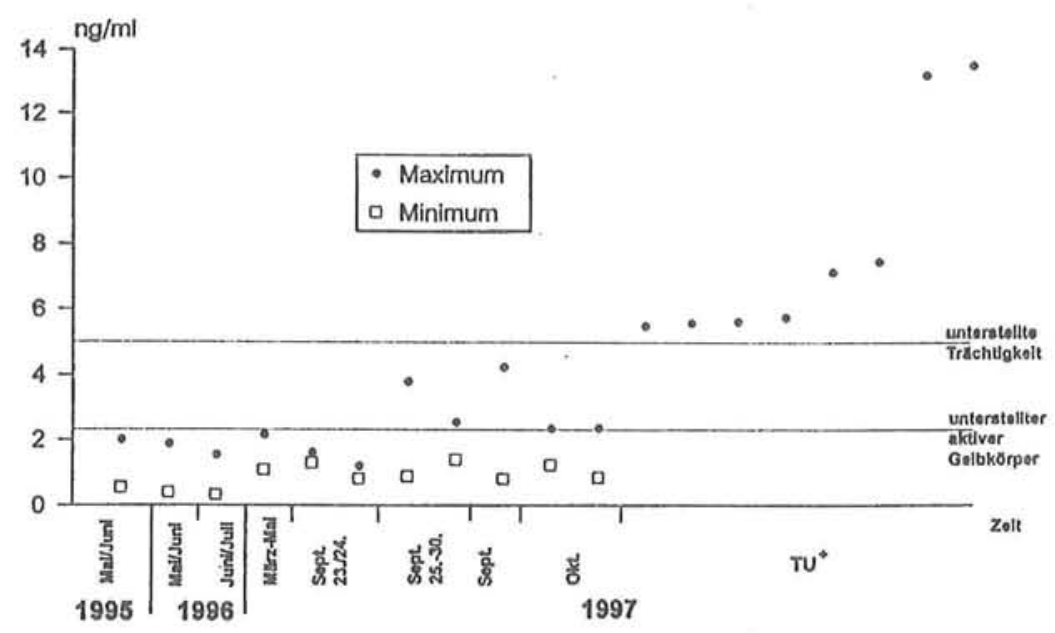

Abb. 2: Progesteron Maximum- und Minimumwerte bei Einzeltieren (Blood progesterone level (minimum and maximum) in single sheeps)

Der Progesteronwert in der Zyklusmitte befindet sich nach BOSTEDT u.a.(1981), SOUZA u.a. (1997) und RAVINDRA (1997) bei $1,88 \mathrm{ng} / \mathrm{ml}$ bis $2,55 \mathrm{ng} / \mathrm{ml}$ Blutserum. In der Nähe der Zyklusmitte wäre ein Ansatzpunkt für eine biotechnische Steuerung, beispielsweise Einleitung der Luteolyse durch Prostaglandin F 2 alpha, gegeben. Der entsprechende Progesteronpeak muß durch Verfolgsblutung ermittelt werden. Die U1traschalldiagnostik am Ovar kann über die Darstellung besonders von Follikeln und ihrer Größenverhältnisse den Verlauf der Hormonkurve untermauern. Da subklinisch mit hoher Wahrscheinlichkeit ein ovarieller Zyklus abläuft, ist das Ultraschallbild eine wertvolle Ergänzung zur Einschätzung eines beginnenden fertilen Zyklus. Aus der Entwicklung der Follikel dürfte sich auch eine weitere Stimulationsmöglichkeit (Ovulationssteuerung) mit GnRH oder Gonadotropinen (LH; HCG) ergeben.

1996 wurde der Zeitraum der Blutentnahme bis Ende Juli verlängert. Die Progesteronwerte verblieben im Mai / Juni auf einem niedrigen Niveau bei einem Minimum von $0,4 \mathrm{ng} / \mathrm{ml}$ und dem Maximum von 1,87 $\mathrm{ng} / \mathrm{ml}$ Blutplasma. Hier besteht eine Ähnlichkeit zu 1995. Im Juli wurden Extremwerte zwischen $0,33 \mathrm{ng} / \mathrm{ml}$ und 1,54 ng/ml Blutplasma registriert, also waren wiederum keine auf einen fertilen Zyklus hinweisenden Progesteronkonzentrationen vorhanden (Abb. 2). Nach SOUZA u.a.(1997) und RAVINDRA (1997) wäre zwar mit den $1,87 \mathrm{ng} / \mathrm{ml}$ in vorliegendem Tiermaterial (Mai/Juni) ein auf eine Zyklusaktivităt hinweisender Wert existent gewesen, aber kli- 
nisch war in den Versuchen kein Brunstverhalten (Suchbock) im Vorfeld nachweisbar. Die beschriebenen Differenzen im Nanogrammbereich sind sehr gering, so daß die Unterschiede in diesem Falle methodisch bedingt sein können. Über die Beziehungen des Auftretens klinisch sichtbarer Zeichen einer zyklischen Aktivität außerhalb der Zuchtsaison und Hormondaten gibt es bislang keine eigenen Erfahrungen.

1997 wurde bereits im März mit den Untersuchungen zum Verlauf der Hormonkonzentration begonnen. Aufgrund eines erweiterten Versuchsprogramms sind sie erst im Oktober beendet worden. Die Progesteronwerte bei den ersten sechs Schafen (März bis Mai) lagen höher als 1996, aber gleichfalls ohne deutliche Differenzen. Der Minimalwert betrug 1,1 ng/ml, der Maximalwert 2,15 ng/ml Blutserum. Die wenig unterschiedlichen Werte sprechen wiederum für eine Nichtaktivität. Das insgesamt höhere Progesteronniveau läßt eher auf einen methodischen Ursprung (Verwendung eines neuen Antikörpers) als auf echte Konzentrationserhöhung schließen.

Die parallel an diesen Schafen erhobenen Ultraschallbefunde der Ovarien wiesen eine Ovaraktivität auf. Es konnten Follikel verschiedener Größen nachgewiesen werden, ohne daß ein klinisch sichtbarer Zyklus (Suchböcke) festzustellen war. Drei Schafe wiesen in zwei bis drei Follikelwellen innerhalb von sechs Wochen Größenunterschiede der Follikel von $2 \mathrm{~mm}$ bis $7 \mathrm{~mm}$ auf (Abb. 3). Die Ultraschallbilder und die Progesteronkonzentrationen deuteten auf eine Ovaraktivität hin. Diese reichte aber nicht zur Ausprägung einer fertilen Brunst.

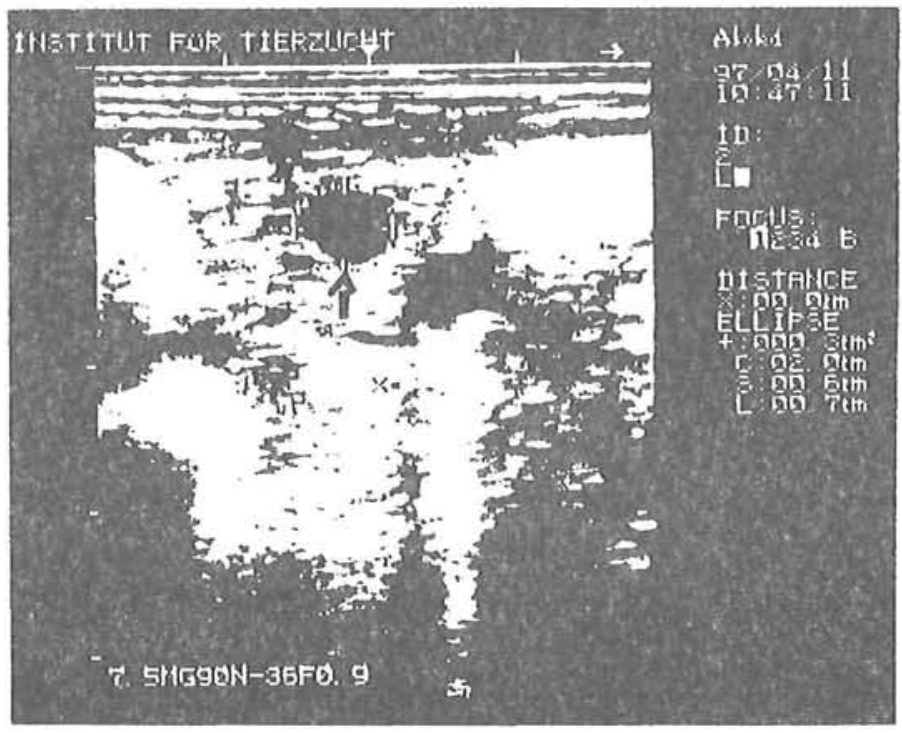

Abb. 3.: Ultraschallbild eines Follikels (Pfeil) im Anöstrus (Ultrasonography of follicle (arrow) on anestrus)

Eine weitere Klärung zum Verhalten der Progesteronkonzentration in einem Zyklus wurde von zwei Schafen über einen Intensivversuch erwartet (Start zu Brunstbeginn). Die über $24 \mathrm{~h}$ kontrollierte Progesteronausschüttung wies bei beiden Tieren ein gleichbleibendes niedriges Niveau mit großer Ähnlichkeit zur Testung im Frühjahr auf. Sowohl die Werte pro Schaf als auch zwischen beiden Schafen differierten nur unbedeutend voneinander (Abb. 2). Diese Konzentration könnte für eine Brunst gewertet wer- 
den, da der Suchbock positiv reagierte. Danach hätten die Schafe vom Frühjahr mit ähnlichen Werten ebenfalls Brunst zeigen müssen, jedoch blieb dort eine klinisch sichtbare Brunst aus (Suchböcke ohne Reaktion).

Eine Erklärung für das Verhalten im Herbst könnte die fortgeschrittene Jahreszeit sein. Die Weiterführung der Progesteronuntersuchung bis Ende September (Abb. 2) ergab bei beiden Schafen einen relativ deutlichen Anstieg auf $3,81 \mathrm{ng} / \mathrm{ml}$ bzw. $2,51 \mathrm{ng} / \mathrm{ml}$ Blutplasma als Maximum. In diesem Zeitraum könnte eine Ovulation und anschlieBende Gelbkörperbildung stattgefunden haben. Die Maxima sind gegenüber vorhergehenden Werten deutlich ausgeprägt und entsprechen etwa den Werten von BOSTEDT (1981) und SOUZA u.a. (1997).

Als möglicher Beweis für eine eingetretene Zyklustätigkeit dürften der zeitliche Eintritt des Folgezyklus und die weitere Entwicklung der Progesteronausschüttung gelten. Beide Schafe erreichten die nächste Brunst spontan nach 15 bzw. 18 Tagen (mit Bedeckung) mit einem Progesteronwert von 1,24 ng/ml bzw.0,87 ng/ml Blutplasma. Innerhalb einer Woche stiegen die Werte auf 2,35 ng/ml bzw. 2,37 ng/ml Blutplasma an. Diese Anstiege könnten in Verbindung mit den Maximalwerten vom vorhergehenden Zyklus und seines Intervalls auf beginnende Trächtigkeiten bzw. einen aktiven Gelbkörper hinweisen.

Zehn dazu parallel auf der Weide von Ende September bis Mitte Oktober getestete Mutterschafe, bei denen ein deckfähiger Bock mitlief, besaßen gleiche Progesteronniveaus wie die vorherigen Testgruppen $(0,81 \mathrm{ng} / \mathrm{ml}$ bis $4,25 \mathrm{ng} / \mathrm{ml})$. Die Einzelwerte entwickelten sich entsprechend den unterschiedlichen Ovaraktivitäten verschieden. Welche Progesteronwerte eine günstige endokrine Voraussetzung für die Entstehung einer Trächtigkeit führten, müssen die Ablammergebnisse belegen. Bei acht bisherigen Ablammungen betrugen die Progesteronkonzentrationen in der ca. 8. Trächtigkeitswoche 5,$50 ; 5,60 ; 5,64 ; 5,76 ; 7,17 ; 7,48 ; 13,25$ und $13,55 \mathrm{ng} / \mathrm{ml}$ Blutplasma.

$\mathrm{Da}$ aus den analysierten Progesteronwerten mit $>2 \mathrm{ng} / \mathrm{ml}$ auf einen aktiven Gelbkörpert geschlossen wird, ist es durchaus denkbar, bei Werten $>5 \mathrm{ng} / \mathrm{ml}$ vom Vorliegen eines Trächtigkeitsgelbkörpers auszugehen. Diese These ist durch einen Folgeversuch zu reproduzieren.

\section{Schlußfolgerungen}

- In vorliegenden Untersuchungen wurde bestätigt, daß die Ovaraktivität beim SKF auch außerhalb der Zuchtsaison nicht erloschen ist.

- Die Zykluskontrolle beim Schwarzköpfigen Fleischschaf mittels Analyse der Progesteronkonzenration ist bedingt möglich. Der Progesteronverlauf ab September läßt den Beginn der Zyklusaktivität erkennen.

- Der erforderliche Aufwand der Probenentnahme und Analyse ist bei orientierenden Untersuchungen über die Situation in der Herde vertretbar.

- Die Wertbarkeit des Progesteronniveaus bis $2 \mathrm{ng} / \mathrm{ml}$ Blutplasma und $>2 \mathrm{ng} / \mathrm{ml}$ als unterstellte Grenzwerte für einen aktiven Gelbkörper muß durch weitere Experimente überprüft werden. 
- Bei beginnender Trächtigkeit ist ein Progesteronanstieg auf $5 \mathrm{ng} / \mathrm{ml}$ und darüber zu erwarten.

- Weitere Forschungsarbeit ist für den Zeitraum Juni / Juli zu leisten und die dort ermittelte ovarielle Aktivität sollte durch biotechnische Maßnahmen zu einem fertilen Zyklus geführt werden.

- Als diagnostischer Parameter für weiterführende Untersuchungen wird die Progesteronkonzentration im peripheren Blut vorgeschlagen. Eine wertvolle Ergänzung kann durch die Ultraschalluntersuchung geleistet werden. Für grundlegende Erhebungen kann darüberhinaus die Analytik des Östradiols bzw. des LH herangezogen werden.

\section{Literatur}

BLÖDOW, G.; GÖTZE, M.; KITZIG, M.; BRÜSSOW, K.-P.; DUSCHINSKI, U.:

Radioimmunologische Steriodhormonbestimmungen in der Follikelflüssigkeit bei Rind und Schwein. Isotopenpraxis 24 (1988), 151-155

BOSTEDT, H.; STOLLA, R.; KLENNER, A.:

Progesteronkonzentration im Blutplasma laktierender und nicht laktierender Schafe in den ersten Wochen nach der Geburt. Zeitschrift Tierzüchtung, Züchtungsbiologie 98 (1981), 11-20

GALLEGOS-SANCHEZ, J.; MALPAUX, B.; THIERY, J.-C.:

Control of pulsatile LH secretion during seasonal anoestrus in the ewe. Reprod. Nutr. Dev. 38 (1998) 3 15

RAVINDRA, J.P.; RAWLINGS, N.C.:

Ovarian follicular dynamics in ewes during the transition from anoestrus to the breeding season. Journal of Reproduction and Fertility 110 (1997), 279-289

SOUZA, C.J.H.; CAMPBELL, B.K.; BAIRD, D.T.:

Follicular dynamics and ovarian steroid secretion in sheep during anoestrus. Joumal of Reproduction and Fertility 108 (1996), 101-106

SOUZA, C.J.H.; CAMPBELL, B.K.; BAIRD, D.T.:

Follicular dynamics and ovarian steroid secretion in sheep during the follicular and early luteal phases of the estrus cycle. Biology of Reproduction 56 (1997), 483-488

Eingegangen: 16.06 .1998

Akzeptiert: 22.02 .1999

Anschriften der Verfasser

VR Dr. sc. med.-vet. FRANK REHBOCK, Dr. habil. WOLFGANG ZUPP, Dr. SVEN GRUMBACH

Landesforschungsanstalt für Landwirtschaft und Fischerei Mecklenburg-Vorpommern

Institut für Tierproduktion

Wilhelm-Stahl-Allee 2 / Haus 9

D-18196 Dummerstorf

Dr. FALK SCHNEIDER

Forschungsinstitut für die Biologie landwirtschaftlicher Nutztiere

Forschungsbereich Fortpflanzungsbiologie

Wilhelm-Stahl-Allee 2

D-18196 Dummerstorf 
Arch. Tierz., Dummerstorf 42 (1999) 2, 190

\section{Buchbesprechung}

Fehlentwicklungen in der Haustierzucht - Zuchtextreme und Zuchtdefekte bei Nutz- und Hobbytieren

THOMAS BARTELS und WILHELM WEGNER

119 Seiten, 40 Abbildungen, 1 Tabelle, Ferdinand Enke Verlag, Stuttgart, 1998, ISBN 3-432-28131-5, 39,80 DM

Das vorliegende Buch beschäftigt sich mit Fehlentwicklungen in der Haustierzucht Ein Thema, das gegenwärtig immer mehr in den Blickpunkt öffentlichen Interesses rückt. Gerade in einer von zunehmender Technisierung bis zur Hochtechnologie geprägten Industriegesellschaft wächst das Interesse vieler Menschen an Haus- und Heimtieren und so wendet sich dieses Buch vordergründig an diesen Personenkreis. Viele sog. Rassetiere wurden zu „Sportartikeln“ oder willkürlich formbaren bzw. deformierten Kunstwerken degradiert. Den tierischen Bedürfnissen entgegenstehende, oft abwegige, Zuchtziele und das Ausstellungswesen mit seinen teilweise bizarren und völlig unbiologischen Standardforderungen hat teilweise zu Mißständen gefuihrt, auf die die Autoren die Aufmerksamkeit des Lesers lenken wollen. Auf solche Fehlentwicklungen aufmerksam zu machen, das Bewußtsein für Zuchtdefekte bei den Haltern, potenziellen Käufern solcher sog. „Rassetiere" zu schärfen, ist Verdienst und vordergründiges Anliegen der Autoren. Gerade bei sog. Rasse-, Hobbyzüchtern und Heimtierhaltern bestehen teilweise erhebliche Defizite an tierzüchterischen, erbpathologischen und nicht zuletzt biologischen Kenntnissen.

In den 5 Kapiteln des Buches wird über sehr zahlreiche Beispiele, überwiegend bei kleinen Haustieren, insbesondere bei Hund, Katze, Hauskaninchen, Huhn, Wassergeflügel, Taube, Ziergeflügel oder Fischen u.a., belegt durch umfangreiche Literaturnachweise, berichtet. Sowohl bezüglich der Literatur als auch der im Buch gewählten Dimensionierung sind erfreulicherweise die Beispiele bei den größeren landwirtschaftlichen Nutztieren gering. Zumal verantwortungsvolle Züchter die durch einseitige Forderungen des Marktes verursachten züchterischen Fehlorientierungen im eigenen Interesse schnell korrigieren. Im Schriftum ist zu belegen, daß diese landwirtschaftlichen Züchter sich stets vom Wort des Aristoteles leiten lassen, wonach das Ganze mehr als die Summe seiner Teile ist. Eine klarere Trennung der Zuchtauffassungen dieser verantwortungsbewußten Nutztierzüchter und der sog. „Rasse- bzw. Hobbyzüchter“ ist im Buch nicht eindeutig erkennbar. Während bei ersteren abiologische Gestaltungen von Selektionsprogrammen langfristig gravierende Auswirkungen für die Ökonomie und den Fortbestand der Population zur Folge haben, erhöhen bei den Hobbyzüchtern, wie im Buch sehr gut dargestellt, diese züchterischen Fehlentwicklungen oft sogar den Marktwert dieser Zuchtprodukte.

Die 5 Kapitel mit ihren zahlreichen Unterabschnitten sind nach den Hauptgruppen bestehender Fehlentwicklungen gegliedert wie Veränderung der Körpergröße und Körpergestalt, Exzessivbildungen des Integuments, Pig. mentierungsanomalien und Verhaltenshypertrophien sowie zentralnervöse Defekte. Im letzten Abschnitt werden Fragen der Öbertypisierung, des Rassenwandels und der Qualzucht diskutiert sowie ein Resümee gezogen. Die Aussagen sind durch zahlreiche Abbildungen untermauert und drucktechnische Hervorhebungen weisen auf besondere Auffälligkeiten hin.

Das sehr umfangreiche Literaturverzeichnis zeugt vom Bemühen der Autoren diese Problematik möglichst umfassend zu dokumentieren. So, wie im Buchinhalt, enthält das Literaturverzeichnis überwiegend Quellen, die sich mit kleineren Haustieren oder Heimtieren beschäftigen.

Den Autoren ist es gelungen zu verdeutlichen, daß die Haltung und Zucht von Heimtieren und Haustieren zu Liebhaberzwecken in der Wohlstandsgesellschaft z.T. zu abiologischen Auswüchsen gefuihrt hat, die es zu beseitigen gilt. Der Zweck dieses Buches, nämlich ein Problembewußtsein bei Züchtern und Haltern zu erzielen, ist erreicht und wird durch sein Spektrum einen großen Leserkreis ansprechen. 